\title{
Faserverbundwerkstoffe für die Großserie
}

Zwar eignen sich endlos faserverstärkte Verbundwerkstoffe mit thermoplastischen Kunststoffmatrizes sehr gut für den Automobilbau. Sie herzustellen ist jedoch umständlich. Eine neue Herangehensweise macht nun den Einsatz des Spritzgussverfahrens möglich. Gemeinsam mit dem Spritzgussmaschinenbauer Engel Austria haben die Forscher des Fraunhofer-Instituts für Chemische Technologie ICT in Pfinztal nun erstmals eine Technik zur Produktreife gebracht, die eine Serienfertigung solcher endlos faserverstärkten thermoplastischen Verbundstrukturen im Spritzgussverfahren erlaubt.

Bislang war es sehr aufwendig, endlos faserverstärkte Verbundwerkstoffe mit einer thermoplastischen Matrix in großen Stückzahlen herzustellen. Zum einen lassen sich textilähnliche dichte Endlosfaserstrukturen nur sehr schwer in Form bringen, zum anderen ist das Zusammenbringen der Endlosfasern mit einem hoch viskosen thermoplastischen Matrixwerkstoff ein sehr komplexer Prozess.
Eine wirtschaftlich rentable Produktionstechnik für große Bauteilserien fehlt deshalb bisher. Es war lediglich möglich, Faserverbundwerkstoffe aus Kurzfasern oder Langfasern zu spritzgießen. "Mit dem Spritzgießverfahren können Bauteile mit einem hohen Faservolumengehalt und daher exzellenten mechanischen Eigenschaften effizient in Serie hergestellt werden", sagt Dr.-Ing. Lars Fredrik Berg, wissenschaftlicher Mitarbeiter und Projektleiter am ICT.

Aufbauend auf den eigenen Forschungsergebnissen haben die Forscher des ICT zusammen mit Engel eine Prototypenanlage für das Spritzgießen entworfen. Diese könne alle notwendigen Arbeitsschritte innerhalb einer Anlage erledigen. Die reaktiven Komponenten würden aufbereitet und vermischt und der Werkstoff in das Formwerkzeug injiziert. Dort finde auch die Polymerisation nach der Infiltration der textilen Verstärkungsstrukturen statt. Der Prozess, der vorher auf mehrere Anlagen verteilt war, sei jetzt auf einer möglich.
Im Unterschied zu bisherigen Spritzgussverfahren für Faserverbundwerkstoffe, bei denen sich lediglich Kurzfasern verarbeiten ließen, kann man den Forschern nach in der neuen Anlange direkt endlos faserverstärkte Verbundstrukturen einlegen und mit einer sehr niedrig viskosen Kunststoffmatrix infiltrieren. Das Verfahren erlaube die In-situ-Polymerisation von thermoplastischen Matrixmaterialien. Dazu würden Monomere direkt in der Anlage polymerisiert. Die reaktive Kunststoffmatrix sei bei der Verarbeitung ähnlich viskos wie Wasser. Somit könnten die Faserstrukturen ideal benetzt werden, ohne die Strukturen in der Form zu verschieben.

Für den Automobilzulieferer ZF Friedrichshafen fertigte Engel bereits exemplarisch einen Bremspedal-Einleger aus glasfaserverstärktem Polyamid. Die Industrievereinigung Verstärkte Kunststoffe (AVK) hat das ICT und Engel für die neue Technik mit dem AVK Innovationspreis in der Kategorie „Verfahren" ausgezeichnet.

NEUES TESTZENTRUM FÜR TRAGSTRUKTUREN

FORSCHUNG ZU OFFSHORE-WINDENERGIEANLAGEN

Im Testzentrum für Tragstrukturen in Marienwerder soll künftig Forschung zu OffshoreWindenergieanlagen betrieben werden. In dem im Bau befindlichen Zentrum mit einer rund $20 \mathrm{~m}$ hohen Versuchshalle wollen Experten aus dem Fraunhofer IWES und verschiedenen Instituten der Leibniz Universität die Bedingungen auf hoher See nachahmen. In einer mit Wasser und Sand gefüllten Grube können originalgetreue Komponenten von OffshoreWindenergieanlagen erprobt werden. Die Belastung, der die Windenergieanlagen im Betrieb auf See ausgesetzt sind, wird durch
Hydraulikpressen nachgeahmt. Ziel der Forschungsprojekte ist es unter anderem, die Lebensdauer von Windenergieanlagen zu verlängern, die Produktionskosten zu senken und die Tragstrukturen der Windenergieanlagen sicherer zu machen.

Das Fraunhofer IWES und die Leibniz Universität haben einen Kooperationsvertrag über gemeinsame Aktivitäten im Bereich der Windenergieforschung unterzeichnet. Die Errichtung des Testzentrums für Tragstrukturen ist ein Teil der Kooperationsvereinbarung. Durch die dort möglichen, realistischen Tests will das
Fraunhofer IWES mit seinen Projekten die Zeit bis zur Anwendungsreife von Tragstrukturdesigns verkürzen.

Das Testzentrum für Tragstrukturen ist mit Gesamtkosten in Höhe von 25 Millionen Euro veranschlagt. Der Bau wird überwiegend vom Bundesministerium für Umwelt, Naturschutz und Reaktorsicherheit finanziert, aber auch das Land Niedersachsen, die Europäische Union und die Leibniz Universität sind beteiligt. Voraussichtlich Ende 2013 soll das Zentrum fertiggestellt sein und bis Mitte 2014 in Betrieb gehen. 